\title{
DESARROLLO Y CONSTRUCCIÓN EXPERIMENTAL DE UNA CUBIERTA TRANSFORMABLE
}

Articulo de Reflexion - Recibido: 20 de Agosto de 2015 - Aceptado: 18 Noviembre de 2015

\author{
Carlos César Morales Guzmán ${ }^{1}$ \\ Universidad Veracruzana. Poza Rica-Tuxpan, México.dr.arqmorales@gmail.com
}

Para citar este artículo / to reference this article:

Morales, C. (2016). Desarrollo y Construcción Experimental de una Cubierta Transformable. Módulo Arquitectura CUC, Vol.16 N¹, 87-118.

\begin{abstract}
Resumen
Mediante la investigación experimental de un sistema plegable se ha desarrollado la estructura de una cubierta transformable. Se han tomado como referencia sistemas ligeros que se caracterizan por formar construcciones versátiles y adaptables. El punto clave de dicho proyecto se materializa con la construcción de un prototipo escala 1:1 mediante el cual se comprueba que puede construirse de manera muy rápida si sus elementos permiten la plegabilidad del sistema. Así pues, con el estudio del prototipo se busca mejorar las uniones y nodos implementados en su construcción. La estructura plegable de la cubierta está constituido por elementos de contra venteo articulados, generando nodos móviles que absorben las fuerzas de presión y succión emitidas por el viento en ambas cara de la estructura, además de que estos le dan mayor estabilidad. El claro efectivo del prototipo es de $25.00 \mathrm{~m}$ por $27.00 \mathrm{~m}$ de longitud y tiene un peralte efectivo de $2.00 \mathrm{~m}$, el cual trabaja como una armadura de alma abierta circular. Lo más importante de este proyecto son los detalles constructivos que fueron diseñados precisamente para optimizar el funcionamiento de todo el sistema. Es importante mencionar que el prototipo está parcialmente terminado.
\end{abstract}

\section{Palabras clave}

Simulación Estructural, Diseño Experimental, Detalles Constructivos, Plegable, Transformable. 


\section{DEVELOPMENT AND EXPERIMENTAL CONSTRUCTION OF A TRANSFORMABLE ROOF}

\section{Abstract}

Through an experimental research, which was focused on a folding system, the structure of a transformable cover has been developed. Lightweight systems have been taken as references which are characterized by establishing functional and adaptable constructions. The focal point of this project is aimed to materialize through the construction of a 1: 1 scale prototype which has showed that it should have built forthwith on the assumption that its elements have allowed the system to be folded. Thus, the study of the prototype it tests different aspects in order to improve the joints and nodes implemented in the mentioned construction. On the other hand, the collapsible structure of this cover is founded by articulated anti-vent elements, by generating movable nodes that absorb the pressure and suction forces emitted by the wind on both sides of the structure, to offer them outstanding stability .Futhermore,the clear prototype effect is $25.00 \mathrm{~m}$ by $27.00 \mathrm{~m}$ in length and has an effective $2.00 \mathrm{~m}$ cantilever, which has worked as a circular open web armor. Finally, it can be said that the relevance of this project are the constructive details that were designed in order to optimize the operation of the whole system and It is significant to mention that the prototype was partially finished.

\section{Keywords}

Structural Simulation, Experimental Design, Constructive Details, Foldable, Transformable. 


\section{Introducción}

Es inminente, que hoy en día las innovaciones tecnológicas han ganado un amplio campo en la búsqueda de nuevas metodologías constructivas que nos permitan generar espacios versátiles y de rápida edificación. Así pues, en la búsqueda de estas nuevas transformaciones del espacio arquitectónico, en el presente trabajo se ha experimentado con modelos plegables de formas básicas.

La morfología conceptual de dichos modelos, a los cuales nos referiremos durante el desarrollo de la investigación, consiste en la segmentación de una figura cilíndrica y una figura esférica.
A la estructuración de estás se le ha añadido el uso de tijeretas plegables, que eventualmente permitirán la generación de una flexibilidad estructural para posteriormente obtener formas más aproximadas a una cubierta cilíndrica. Este diseño es constituido a partir de iteraciones de múltiples geometrías, pero que para el objeto de este trabajo solo serán utilizadas para generar formas plegables básicas.

Con el objetivo de explicar de una manera más clara se experimentará con tres modelos de tipo tijereta. Cabe mencionar que cada uno de los modelos experimentales está basado en principios matemáticos y graficados en base a su figura matemática.

Tabla 1

Formas básicas para generar una estructura plegable.

Sistema de Tijera

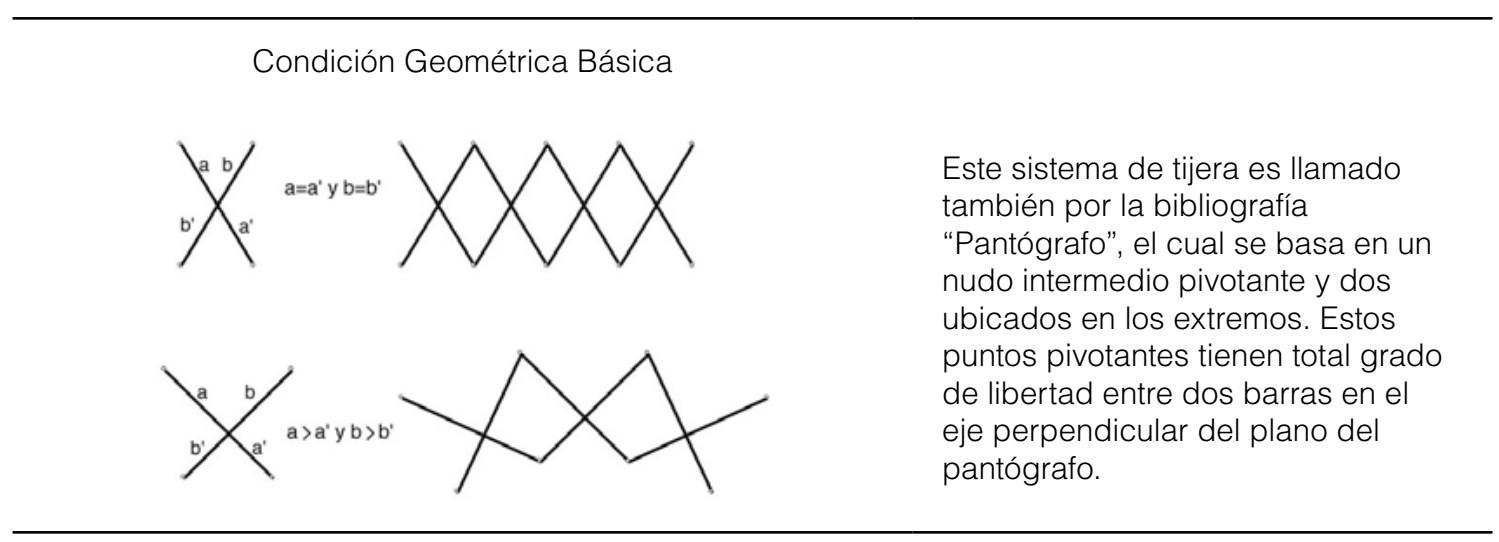


Para una mejor comprensión de este tipo de estructura, se generó una tabla con los principios básicos y características con los que debe contar cualquier estructura geométricamente plegable (Tabla 1). Cabe mencionar que dichos principios y características deben ser contemplados dentro del sistema para lograr la flexibilidad estructural deseada. Posteriormente se podrá realizar la traslación geométrica matemática o descriptiva de la estructura con la que se experimenta y de esta manera generar una serie de iteraciones y modulaciones, que conducirán a una serie de propuestas geométricas.

Para determinar el modelo final de las experimentaciones geométricas, a continuación se realizará la modelación de un sistema cuyo análisis se hace en la siguiente etapa de la investigación. En dicha propuesta formal se aplica la teoría expuesta anteriormente, la cual nos da las pautas para desarrollar un sistema con la suficiente eficiencia estructural para su aplicación, siendo la plegabilidad retráctil su principal característica. A partir de esto, deducimos que el factor determinante es generar un nodo que sea capaz de permitir que la estructura pueda plegarse sencillamente pero que también pueda soportar las tensiones externas que se originan por el constante movimiento al que estarán sujetos cada uno de los elementos del sistema.

A fin de darle solución a las problemáticas mencionadas se experimenta el diseño del nodo. El nodo debe ser muy puntual, es decir, las partes que lo constituyen no deben tener excentricidad al momento de conectarse, ya que cualquier desfase provocaría que la geometría actúe de una forma distinta a la que hemos proyectado en los anteriores ejemplos. La búsqueda de un diseño mejorado de este tipo de uniones, da pie a la búsqueda de más posibilidades tecnológicas. No obstante que las las estructuras transformables son muy poco utilizadas debido a que su manufactura es muy compleja, el objetivo del presente proyecto es la de realizar una unión lo más acorde y sencilla dentro de las posibilidades para la construcción de dicho sistema.

A continuación efectuaremos nuestro primer modelo experimental. Este consiste en la generación de nodos cuadrados con placas situadas simétricamente en sus ejes que formarán una cruz perfecta 

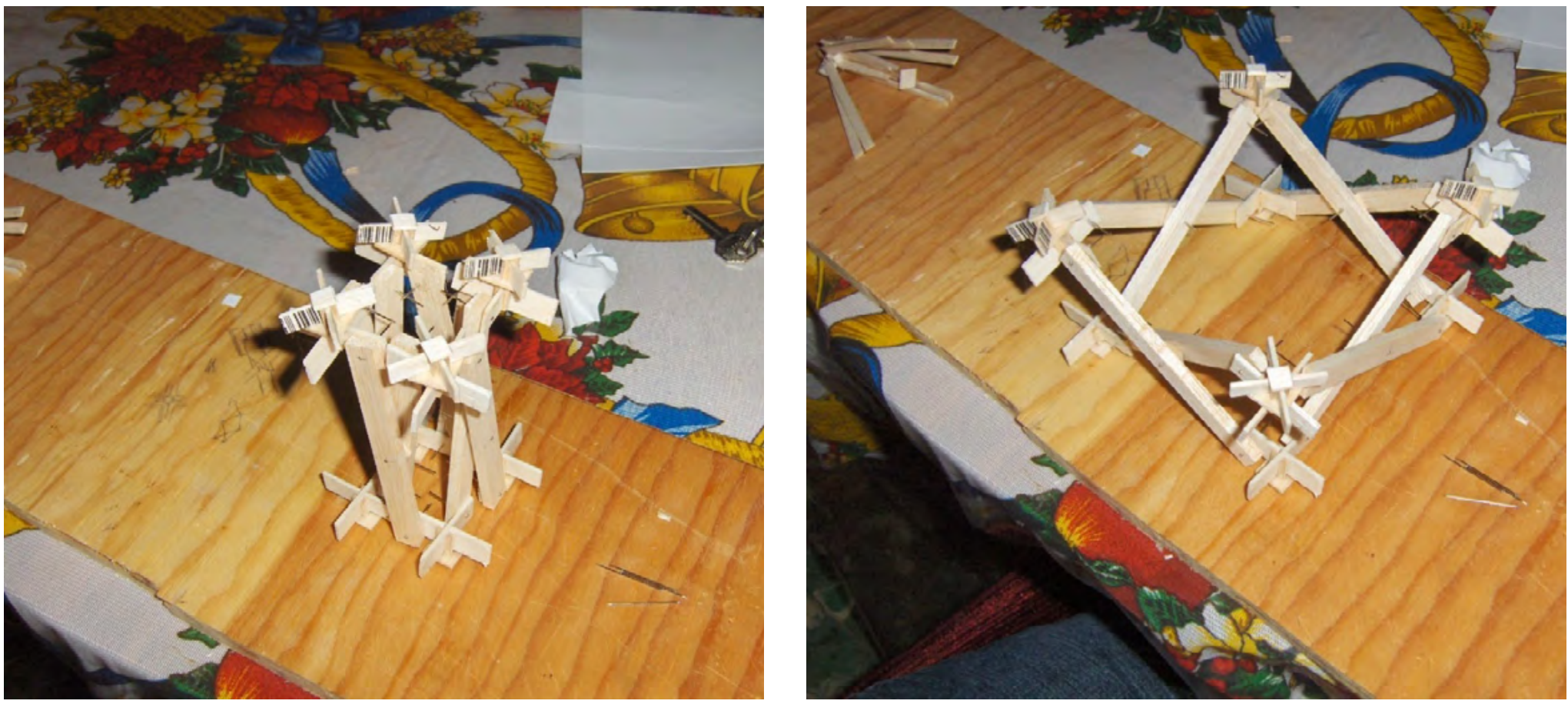

Figuras 2 - 3. La experimentación nos ayuda a generar los parámetros de diseño, para construir el modelo correcto de la geometría plegable, además de que nos permite verificar los errores posibles del sistema. Fuente: Morales, (2010).

(Figuras 2 y 3). La parte superior del nodo está constituida con otro accesorio que de igual manera está hecha de placas y forma una cruz pero esta vez girada a $45^{\circ}$. En la parte inferior el nodo es más sencillo y su función solo es el de sostener y conectar los elementos en la cruceta. Es importante mencionar que la parte superior del nodo ubicado arriba, se encuentra en un ángulo de $45^{\circ}$ ya que es una cúpula rebajada lo cual quiere decir que sus cruces nodales pueden unirse en un solo punto que los converge.

Siguiendo con el modelado, (figuras 4 y 5) se une los elementos de contra venteo con un nodo de placas a ejes formando una cruceta más sencilla. Esta unión es importante ya que mantiene la estabilidad en ambos sentidos de toda la estructura, 

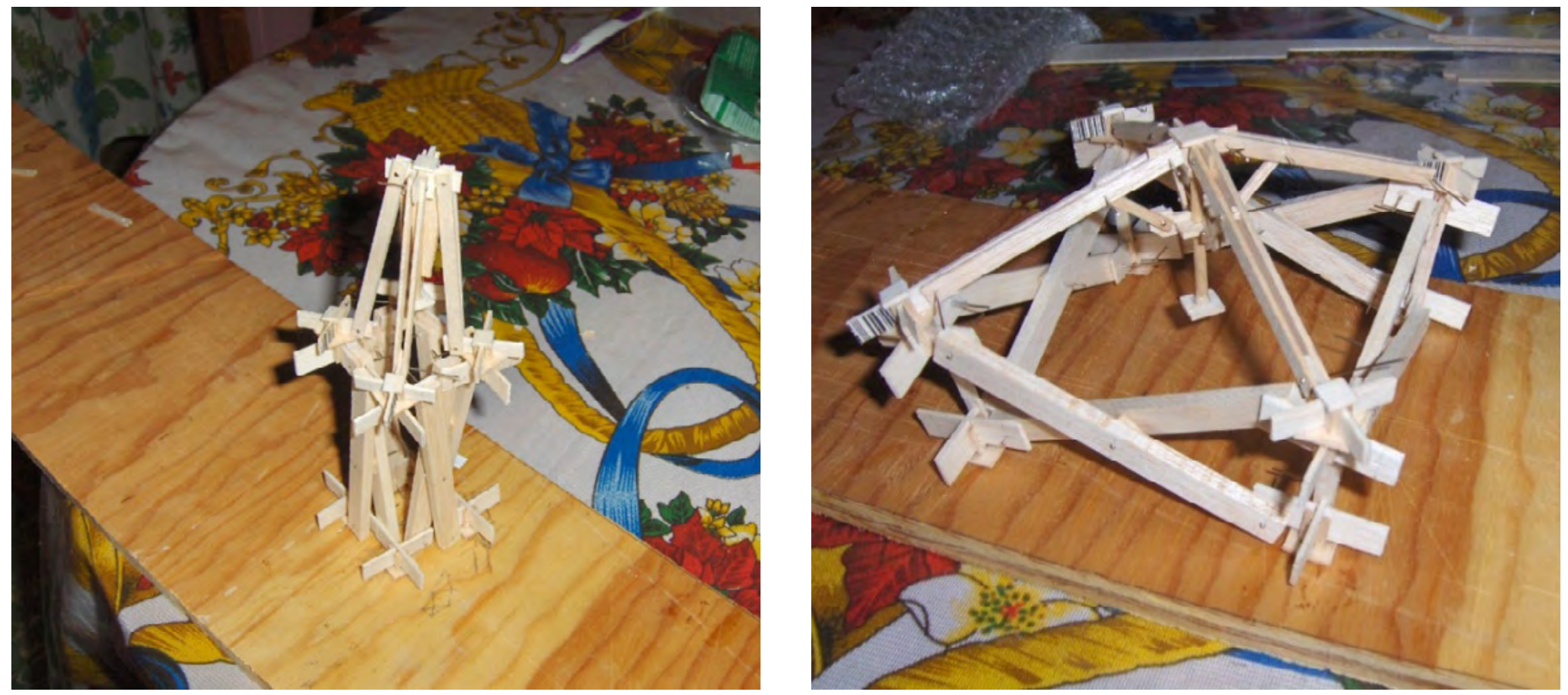

Figuras 4 - 5. Las conexiones de contra venteo son los más difíciles de elaborar ya que suelen tener ángulos de conexión muy cerrados.

Fuente: Morales, (2010).

además de que reparte las fuerzas de presión que pueden llegar a generarse sobre ella. A esta conexión se le aplicó experimentalmente una unión tipo paraguas y se observó que esta le da mucha más versatilidad y estabilidad a la estructura; sin embargo dicho ejercicio se dejara para otro tipo de proyectos. En este caso solo se trabajará con la conexión de contra venteo a ejes para unión de los vanos que forma la cuadricula geodésica esférica.

Posteriormente se realiza el marco de conexión. Este marco es el que absorberá todos los esfuerzos originados estáticamente dentro de la estructura y los repartirá hacia los apoyos. En este sentido, teóricamente se encuentran simplemente apoyados y articulados para una mayor movi- 

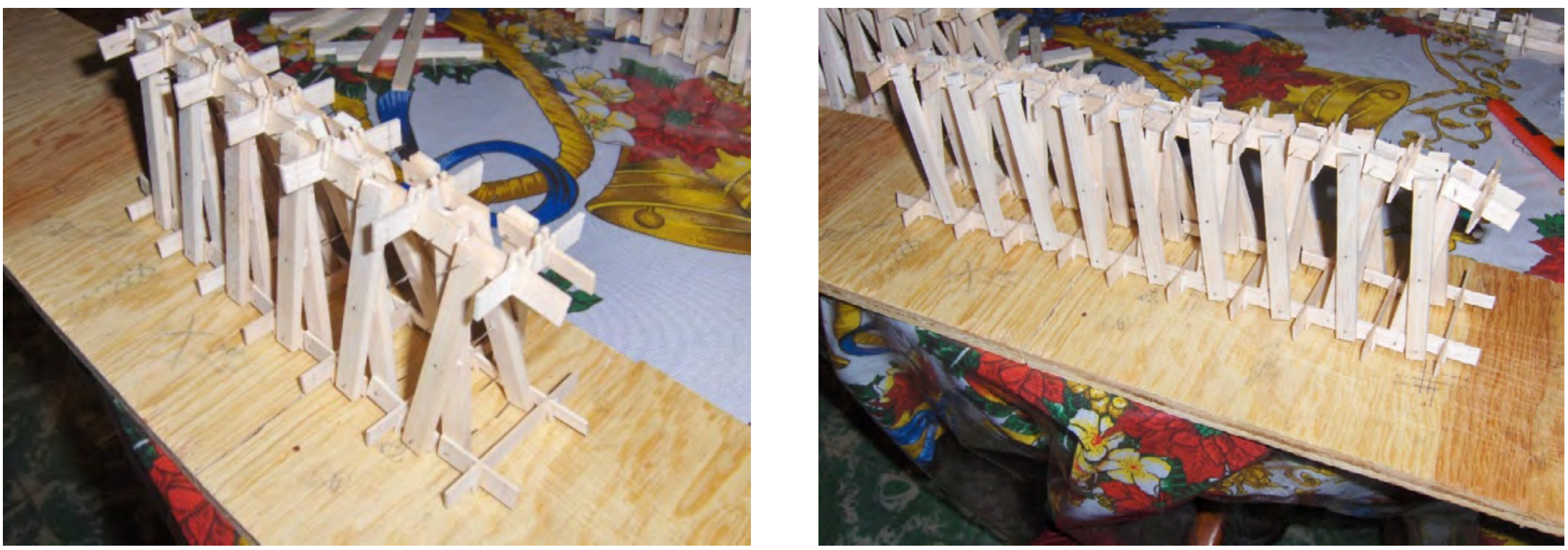

Figuras 6 - 7. El marco de alma abierta se ha elaborado con combinaciones de ángulos y nodos. Este es una conexión importante para el presente proyecto de investigación, ya que resuelve la estabilidad de la geometría.

Fuente: Morales, (2010).

lidad estructural (Figuras 6 y 7). En este modelo plegable, la hipótesis es que debe funcionar como una cubierta de vigas de alma abierta en la que su función es mucho más eficiente si sus nodos son rigidizados con la cubierta. Antes de adentrarnos en el tema de la cubierta, describiremos la viga de marco estructural con el fin de una mejor comprensión de su sistema. Esta se compone de dos tipos de nodos. El primero, que se encuentra en la parte superior, es pequeño y está integrado por placas a ejes en un sentido longitudinal y en el transversal las placas se colocan en un ángulo de $27^{\circ}$ para que los elementos en cruceta puedan situarse en el ángulo correcto de plegado. El segundo nodo, que está en la parte inferior está compuesto por un miembro alargado longitudinalmente y por placas en un ángulo de $27^{\circ}$ en el sentido transversal. Así pues, se logra formar una figura triangular que le da estabilidad propia al marco de alma abierta. 

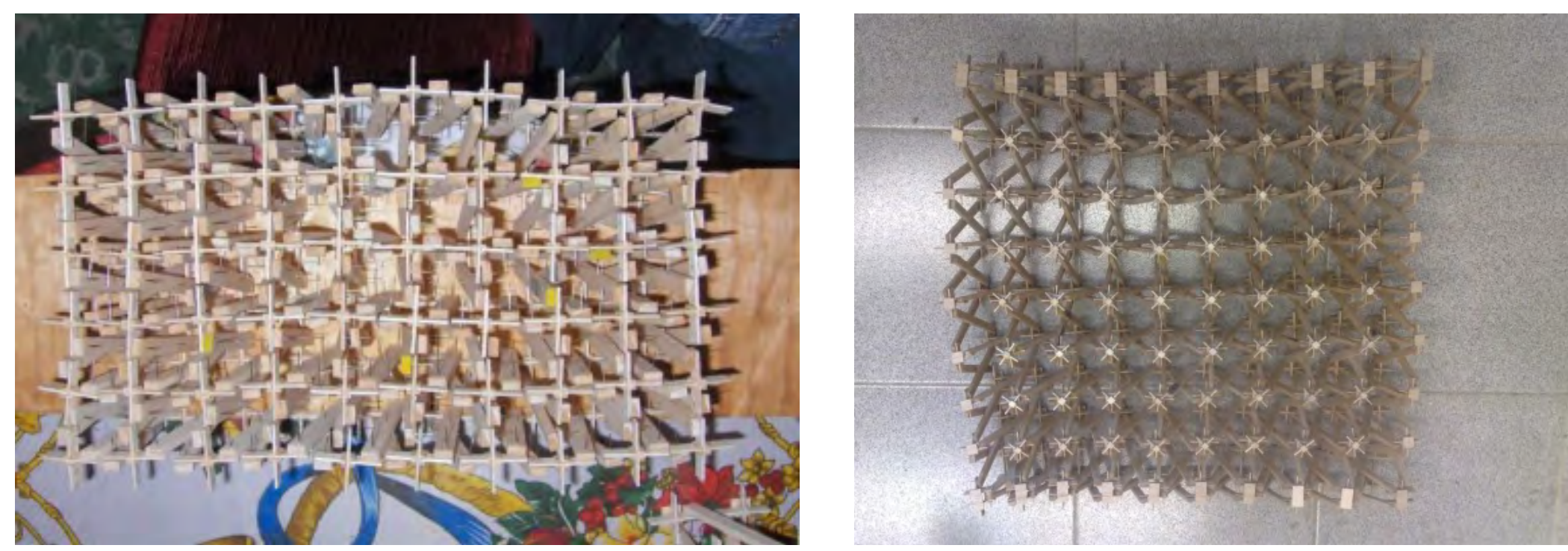

Figuras 8 - 9. La cubierta, ya integrada con las conexiones necesarias, funciona como una superficie activa de alma abierta y redistribuye perfectamente los esfuerzos en la estructura.

Fuente: Morales, (2010).

Finalmente, la cubierta se realiza en base a la composición de la primera etapa del modelo. Esto es mediante su reproducción a lo largo de toda la cubierta formando el casquete esférico de la nave (Figuras 8 y 9). Las partes de este modelo se fueron revisando paso a paso hasta que se notó que los nodos de contra venteo y los nodos de conexión de contra venteo con el nodo de la parte superior de la estructura no siempre coincidían en sus ángulos de unión. Esto en el proyecto final puede tradu- lo más catastrófico), por lo que debe tomarse en cuenta en su ejecución real para que no presente los mismos problemas constructivos.

Consecuentemente de la unión de las partes del sistema se realizara la forma de la cubierta que se encuentra perfectamente equilibrada. La importancia de la experimentación es muy importante, ya que se detecta de una manera eficaz los posibles defectos que puede llegar a presentar la estructura. Además se comprueba que se abre perfectamente cuando no tiene los contramarcos (Figuras 10-12) 

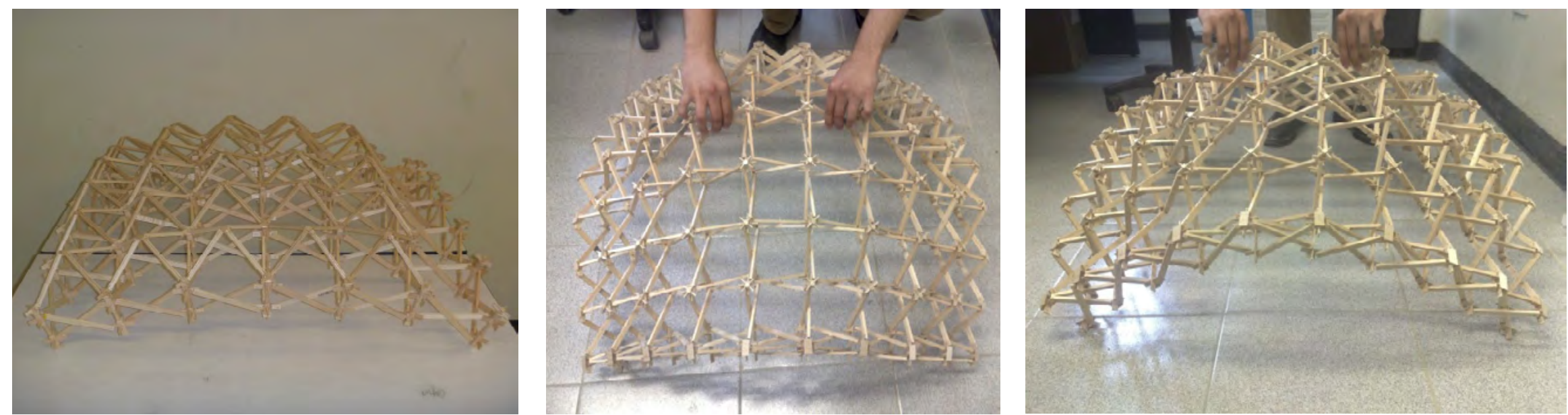

Figuras 10 - 12: La cubierta integrada con las dos partes tiene una función estructural estable, sin embargo las conexiones de contra venteo rigidizó en demasía las conexiones.

Fuente: Fotos Experimentales, Dr. Morales, (2010).

pero sin embargo en el momento de conectar los contra venteos no cuenta con la misma plegabilidad que antes. Esto se debe a que la estructura no se encuentra geometrizada adecuadamente con los elementos de contra venteo que forman los marcos estabilizadores dando como resultado una rigidizarían excesiva en los vanos de la estructura.

A partir del análisis de las anteriores experimentaciones, podemos desarrollar un modelo más aproximado en una escala 1:20. Las dimensiones para la estructura final deben ser para su claro transversal de $25.00 \mathrm{~m}$ y $27.50 \mathrm{~m}$ en el longitudinal.

\section{Simulación de Modelos Constructivos}

Para concluir la etapa de experimentación y construcción de una propuesta de diseño estructural de un sistema transformable, a continuación se presentan dos propuestas de modelos constructivos simulados por computadora.

El programa utilizado para la simulación, ejecuta un cálculo de la conexión y explora el diseño de unión eficiente y funcionalmente estructura para así poder distribuir adecuadamente los esfuerzos que actúan en ella. Así podremos explorar las cualidades, ventajas y desventajas de cada uno 


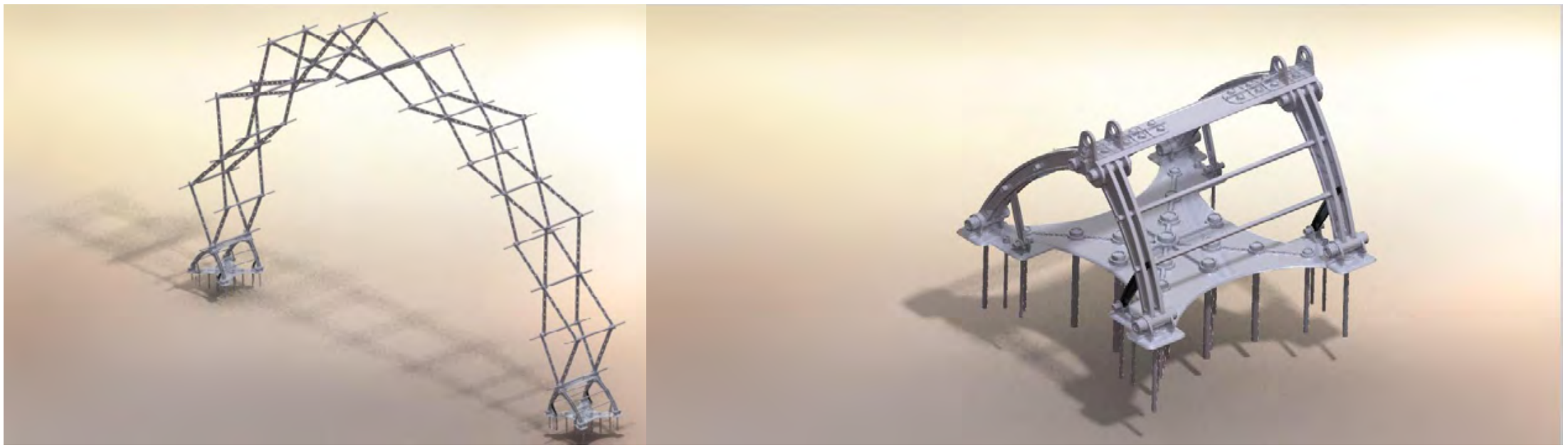

Figuras 13 - 14. Desarrollo experimental de la estructura plegable con soporte orgánico rígido.

Fuente: Morales, (2010).

de los modelos. Dichas conexiones fueron pensadas y diseñadas para construirse con acero estructural A36 o denominación europea S23, puesto que con estos parámetros se han diseñado detalles básicos de unión.

La importancia de dicha exploración de la conexión mediante el programa de diseño, es que nos marcará parámetros para la construcción de un prototipo escala 1:1, además de que permitirá verificar paralelamente aspectos como el montaje y comportamiento estructural.

Recordemos que las propiedades 96 tiene presentar excentricidad alguna. Recordemos que en el modelo experimental anterior se propusieron elementos cuadrados y rectangulares, que en conclusión no funcionan para nuestro prototipo. Esto debido a que la geometría de dichas figuras cuenta con un radio de giro muy amplio, anulando su excentricidad al momento de acomodar sus caras. Ante esto, se propondrán otro tipo de elementos como solución.

\section{Propuesta 1}

Estructura Retráctil Unidireccional compuesta de miembros y accesorios lineales sin nodos (Figuras 13 y 14). 


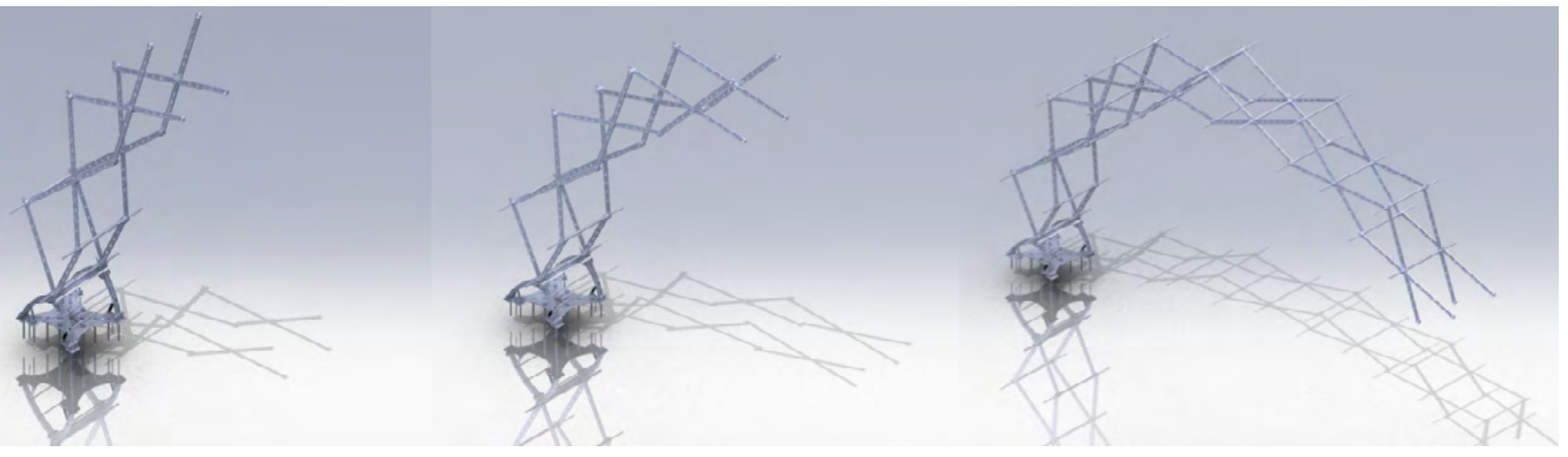

Figuras 15 - 17. Proceso de despliegue y montaje de la Estructura Transformable Unidireccional. Fuente: Morales, (2010).

\section{- Descripción}

La estructura está constituida por elementos de forma rectangular con uniones de placa de acero redondeado. Sus conexiones están sujetas por bushing de articulación y col roll, que permite que la estructura pueda plegarse en un sentido. La sujeción está conectada con tubos de acero en posición horizontal, el cual ayuda a rigidizar en sentido longitudinal la estructura. La característica principal en el diseño de esta estructura es que cuenta con una conexión articulada colocada en posición curvada en su basamento con la intención de que absorba todo el esfuerzo originado por el movimiento de la estructura. Además también está diseñado para resistir los empujes de la misma acción de cargas que estará expuesta el sistema estructural de la cubierta.

\section{- Tipo y Proceso de Montaje}

Debido a que el movimiento de sus elementos permite una fácil manipulación, esta estructura plegable nos permite instalar con mayor velocidad la cubierta. Se plantea que durante el proceso de construcción, la estructura se pueda izar con la asistencia de una grúa o mediante el armado por partes de los mismo elementos. El segundo procedimiento puede llevar más tiempo y lo que se busca es la velocidad de montaje (Figuras 15 - 17). 


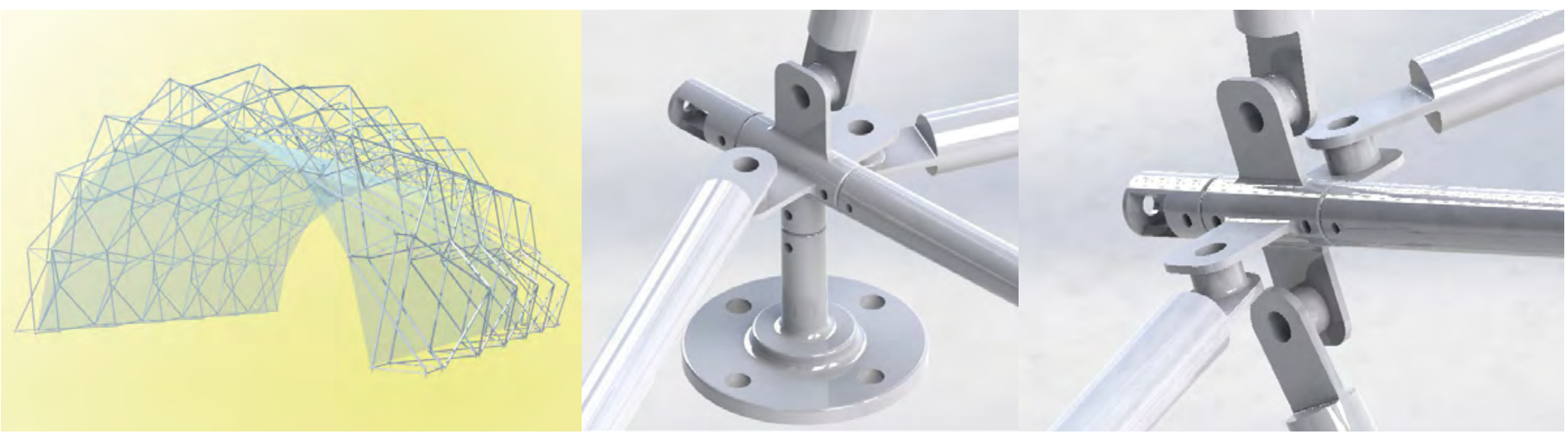

Figuras 18 - 20. Mejoramiento de Uniones de Cubierta Plegable Unidireccional.

Fuente: Morales, (2010).

\section{- Ventajas}

Esta estructura plegable permite que la construcción de la cubierta deseada sea más rápida, además de ser mucho más ligera y esbelta que cualquier otro sistema. Por otro lado, ya que sus uniones no cuentan con elementos rigidizadores verticales, puede absorber y redistribuir con mucha facilidad las cargas horizontales de la estructura al basamento permitiendo el equilibrio total del sistema. También tiene la ventaja de que su construcción resulta poco compleja pues tiene pocos accesorios de conexión.

\section{- Desventajas}

A pesar de que el material utilizado para su construcción es más resistente y ligero que el de otros sistemas, en conjunto con todos sus elementos, la estructura puede llegar a pesar mucho. Sin embargo, la versatilidad de este sistema dependerá de que tan rápido pueda construirse. Por otro lado, el diseño de sus elementos es muy complejo y las conexiones no son del todo excéntricas lo cual podría tener como consecuencia que se tengan que realizar nuevamente su cálculo y rediseñar toda su geometría.

\section{Propuesta 2}

Cubierta Retráctil Unidireccional Cilíndrica con Nodos Flexibles $y$ Articulados en sus extremos (Figuras 18-20). 


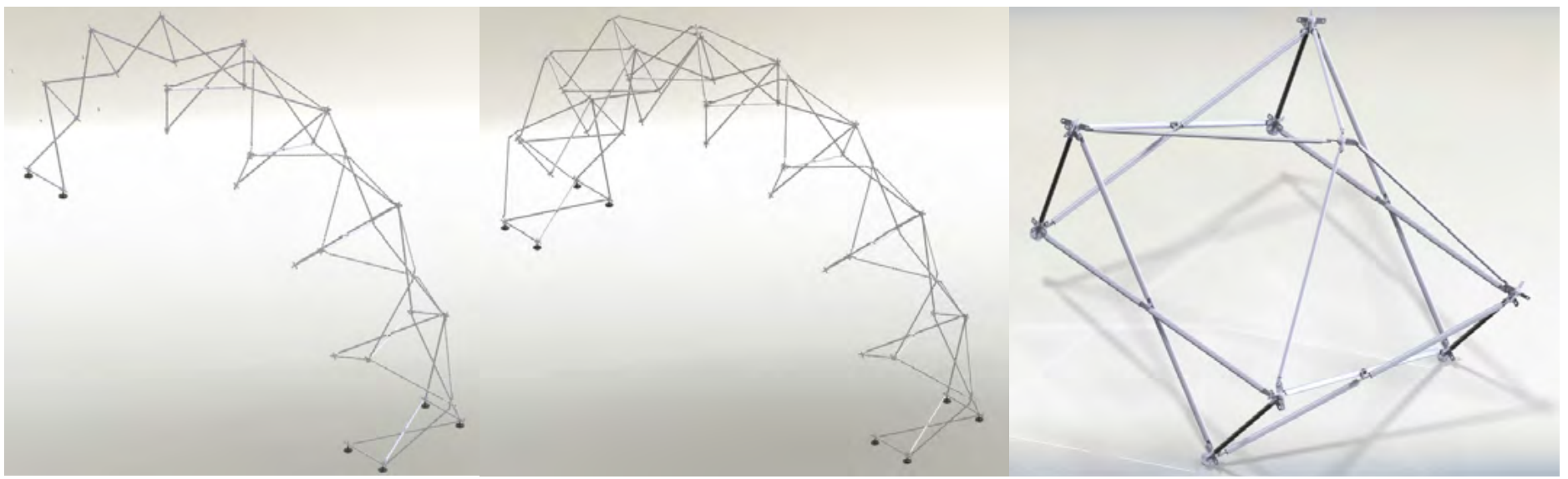

Figuras 21 - 23. Proceso de despliegue y montaje de la Estructura Transformable Unidireccional con contra venteos Fuente: Morales, (2010).

\section{- Descripción}

La cubierta cilíndrica unidireccional está compuesta por nodos flexibles de acero. El nodo se diseñó de la forma más sencilla para poder hacer las conexiones lo más cercanamente posible a la unión. La unión de forma tubular permite una perfecta conexión entre los elementos. Se desarrollaron otros nodos para cada conexión variable que tendrá la cubierta, con esto se le da mayor versatilidad al sistema, por lo que la estructura está diseñada para que pueda transformarse las veces que sea necesaria.

El nodo se ha diseñado de una forma tan versátil que se le pueden integrar infinidad de accesorios para una ejecución exitosa del sistema. Para el nodo se propusieron tubos redondos ya que el radio de giro es más amplio, dando una mayor excentricidad en los elementos ya colocados. A diferencia de la anterior propuesta, el diseño de dicho nodo da la posibilidad de que las fuerzas que actúan en las conexiones actúen de una manera menos agresiva en la estructura. Por último, para que la estructura se integrara en un solo sistema y no tuviera la necesidad de desmontar ningún elemento por separado, se agregó un nodo de contra venteo. Esto último ha mejorado el proceso constructivo de la cubierta. 
- Tipo y Proceso de Montaje

El proceso constructivo y montaje es de fácil ejecución si se usa una grúa para desplegarla. Ya que sus nodos articulados le permiten cierta flexibilidad, también puede ser armada por partes (Figuras 21-23).

\section{- Ventajas}

Una de las grandes ventajas de este sistema estructural, es que al contar con nodos articulados y flexibles, los tiempos de construcción de la cubierta son más cortos y eficientes. Además las conexiones y accesorios entre los nodos son más simples de manipular.

\section{- Desventajas}

Ya que el conjunto de elementos que conforman la estructura puede alcanzar un peso grande, puede presentar dificultades durante las maniobras de desplegado y construcción de la cubierta.

\section{Análisis Estructural}

A continuación realizaremos el análisis estructural para la cubierta ximado de la cubierta y de acuerdo a las recomendaciones del manual del Instituto para la Construcción Tubular $(\mathrm{ITC})^{2}$, se debe dividir la luz entre el $15 \%$ de la luz total del claro para el aprovechamiento máximo de la resistencia y estabilidad estructural. La distancia máxima entre los nodos es de $6.00 \mathrm{~m}$ para que el sistema estructural no pierda el equilibrio. Ya que los cordones superiores e inferiores estarán sujetos a fuerzas de flexión (principalmente el cordón superior) deben ser de una resistencia superior a las diagonales con una influencia de $3,550 \mathrm{~kg} / \mathrm{cm}^{2}$.

Por otro lado, en la estructura de nuestro caso de estudio existirán montantes verticales que ayudarán a soportar las fuerzas de flexión sobre los cordones, mitigando además las cargas axiales de las diagonales, por lo que se consideran los coeficientes del ITC. Sin embargo, como en nuestro modelo son circunferencias, no existirán pilares que las sostengan como en el caso de una viga en celosía. Nuestra estructura se apoyará sobre si misma por lo que no se tiene que

2 Este instituto está especializado en las construcciones tubulares, y sus normas y parámetros se encuentran certificados para su aplicación. www.ictubular.es 
contemplar la translacionalidad ${ }^{3}$ de alfa crítica y ya que su pendiente es superior al 10\%, se tomará el coeficiente de pandeo de 1. Para la mejor comprensión de todos estos requerimientos, se realizarán ejercicios de pre dimensionado y coeficientes de seguridad aplicados para el sistema estructural.

El análisis estructural del proyecto se basa en el rango de factor de seguridad por resistencia y desplazamiento del LRFD4 ${ }^{4}$. Este toma un rango de seguridad de los elementos estructurales de 1 a 1.05 de resistencia del material (acero). La relación de esbeltez $\mathrm{KL} / \mathrm{r}$ es de 240 para elementos principales y de 300 para los elementos secundarios y de contra venteo; la combinación de carga para el sistema se manejará el mismo código de resistencia y estabilidad. También se considera el desplazamiento horizontal y vertical para que no exceda el límite de servicio estructural establecido en el reglamento del Distrito Federal. Adicionalmente, se verán todos los gráficos de tensión para verificar dónde hay mayores esfuerzos

\footnotetext{
3 La translacionalidad e instranslacionalidad, es la que determina el pandeo critico de un pilar sometido a fuerza axial de compresión o cargas de horizontales que hacen que entre en flexión. Con ellos se le aplica el método de Wood que determina el alfa crítica del pilar.

4 Load Resistance Factor Design. (McCormac, 2000).
}

y cómo éstos interactúan dentro de la estructura. Posteriormente, con esta información se desarrollarán las uniones adecuadas para los nodos con la resistencia a estos esfuerzos.

Para determinar la solución a la estabilidad de la estructura se planteará un ejemplo de traslación geométrica y esta luego se analizará por medio de una simulación estática, generando de esta forma los primeros indicios de la tecnología estructural. En dicho ejemplo, tomamos el último de los anteriores ejercicios, ya que su geometría es la menos compleja para realizar este análisis. Recordemos que esta figura geométrica se equilibra y sostiene por si sola ya que está conformada por una serie de marcos plegados en forma de " $X$ ".

En esta simulación se especificó que el material a utilizar sería acero A36 con un módulo de elasticidad de $2,530 \mathrm{~kg} / \mathrm{cm}^{2}$ y un factor de Poisson's ${ }^{5}$ de 0.3 con densidad 7.83847 Mton/m. Las piezas seleccionadas es de tubos

5 El coeficiente de Poisson's se define como una constante elástica del material, que proporciona una medida del estrechamiento de sección de un prisma de material elástico lineal e isótropo cuando se estira longitudinalmente y se adelgaza en las direcciones perpendiculares a la de estiramiento. (Segui, 2000). 
OCXE $100^{6}$ para los elementos de las uniones principales. Los atiezadores son del mismo material y denominación, y para las armaduras plegables en el claro más grande se proponen las mismas piezas tubulares con el objetivo de no complicar tanto el análisis.

Por ende, el resultado será una guía para el comportamiento de los esfuerzos en la estructura; la simulación estática, nos brinda una visión más clara de las dimensiones que pueden llegar a tomar estas adecuaciones geométricas, así como de cual será el claro máximo de la cubierta.

Cabe mencionar que se tomarán las cargas dictadas por el reglamento $y$ el material escogido experimentalmente. La investigación no destina ninguna función espacial a la estructura, ya que el modelo no cuenta con ninguna función en específico. Estos parámetros se toman en cuenta dependiendo de la localización del proyecto, pero para este caso, se determinará la localización en la ciudad de Poza Rica, Ver, y una función espacial de nave industrial, determinando los siguientes parámetros:

6 Miembros Tubulares estándar con nomenclatura OCXXE de acero. Son fabricadas en México para distintas aplicaciones en la construcción de estructuras metálicas. Imca - Instituto Mexicano de la Construcción en Acero. (2005)
Por reglamento de $\mathrm{NCRCDF}^{7}$ las cargas asignadas son; Carga Muerta: $30 \mathrm{~kg} / \mathrm{m}^{2}$, Carga Viva Máxima: $40 \mathrm{~kg} / \mathrm{m}^{2}$ y Carga Viva Accidental: 20 kg/m².

Posteriormente se introducen las cargas anteriores asignadas en el programa Cypecad, las cuales son por reglamento de NCRCDF; Carga Muerta: $30 \mathrm{~kg} / \mathrm{m}^{2}$, Carga Viva Máxima: $40 \mathrm{~kg} /$ $\mathrm{m}^{2}$, Carga Viva Accidental: $20 \mathrm{~kg} / \mathrm{m}^{2}$. Una vez realizado este, la carga horizontal de sismo se hará como cargas externas nodales, y las presiones de viento cambiarían un poco, ya que en cada país se tiene su propio código de aplicación de carga. Para ello solo utilizaremos, la velocidad real básica del viento que para nuestro caso se dio así:

\section{Velocidad de diseño}

La velocidad de diseño, que en este caso resulta constante en todo lo alto de la estructura, es (inciso 4.2):

$V_{D}=0.9(0.86)(150)=116.1 \mathrm{~km} / \mathrm{h}$

7 (NCRCDF) Normas Complementarias de Reglamento de Construcción del Distrito Federal. Arnal y Betancourt (2008). 


\section{Presión dinámica de base}

Dado que el sitio en donde se desplantará la estructura, está prácticamente al nivel del mar, la presión barométrica que le corresponde es de $760 \mathrm{~mm}$ de Hg (Tabla 4.2.5). Además, la temperatura anual media en este sitio es de $24.4^{\circ} \mathrm{C}$. Por tanto, el factor $\mathrm{G}$ vale:

$G=0.392 / 273+\delta$

$\Omega=$ Presión barométrica en $\mathrm{mm}$ de Hg. (Tabla I.7)

$\zeta=$ la temperatura anual en $\mathrm{C}^{\circ}$ (se obtiene en usar las tablas de medida de $\mathrm{C}^{\circ}$ ).

$$
\begin{aligned}
G= & 392(760) / 273+(24.4)= \\
& 297.92 / 297.4=1
\end{aligned}
$$

Puesto que la altura de la estructura es menor que $10 \mathrm{~m}$, la presión dinámica de base es constante en toda su altura. Así, según el inciso 4.2.5:

$q z=0.0048(1.0)(115.1)^{2}=64.7 \mathrm{~kg} / \mathrm{m}^{2}$

Ya que WinTess no realiza métodos de iteración por fuerzas desequilibradas para estructuras de segundo orden y no formas de iteración por combinaciones de carga, colocaremos las cargas respecto a la combinación más pesada y solicitada. Las cargas propuestas para dicho programa son

\begin{tabular}{|c|c|c|}
\hline \multicolumn{3}{|c|}{ WinTess } \\
\hline \multicolumn{3}{|c|}{$\begin{array}{c}\text { Combinación de Carga : } \\
1.4+1.5+\text { SISY }+1.1 \text { VIEN } X+1.1 \text { VIEN Y }\end{array}$} \\
\hline \multicolumn{3}{|c|}{$\begin{array}{c}\text { Sobrecarga }=20 \mathrm{~kg} / \mathrm{m}^{2}+\text { Carga Accidental: } \\
40 \mathrm{~kg} / \mathrm{m}^{2}\end{array}$} \\
\hline $\begin{array}{l}\text { Viento } X= \\
116.1 \mathrm{~km} / \mathrm{h}\end{array}$ & $\begin{array}{l}\text { Viento } Y= \\
64.7 \mathrm{~km} / \mathrm{h}\end{array}$ & $\begin{array}{l}\text { Viento total = } \\
132.9109 \mathrm{~km} / \mathrm{h}\end{array}$ \\
\hline \multicolumn{3}{|c|}{ Tipo de edificio $=$ Abierto $(3)>\operatorname{Sin} c=0$} \\
\hline \multicolumn{3}{|c|}{ Pretensado de la membrana $=0.08 / 0.08 \%$} \\
\hline \multicolumn{3}{|c|}{ Peso de los cables $=791.8 \mathrm{~kg}$} \\
\hline \multicolumn{3}{|c|}{ Peso de los tubos $=35376.0 \mathrm{~kg}$} \\
\hline \multicolumn{3}{|c|}{ Peso de la membrana = $803.7 \mathrm{~kg}$} \\
\hline
\end{tabular}
las siguientes:

A continuación se realiza el proceso de iteración del cálculo. En dicho proceso se desarrolla una matriz de nodos por fuerzas desequilibrantes, es decir, el programa reproduce "n" número de iteraciones hasta lograr equilibrar la estructura. Esto dependerá de la complejidad geométrica de la estructura. Es importante mencionar quemedianteestecálculoobtendremos la dimensión real de los elementos que conformaran la estructura (cables, tubos estructurales), así como los esfuerzos y deformaciones que podría presentar el sistema (ya incluida la membrana). 
Las especificaciones para los elementos de la cubierta plegable son los siguientes: para el caso de la membrana se ha seleccionado una membrana Serge FerrariFluitop-T2-1002 con una resistencia RK (daN $/ 5 \mathrm{~cm}$ ) 420/420; tubos circulares de acero de espesor $150 \mathrm{~mm}$ con denominación europea S235; y cables de acero galvanizado de $40 \mathrm{~mm}$ y $32 \mathrm{~mm}$ de espesor. Una vez realizado el cálculo bajo estas especificaciones se verifican los datos arrojados por el programa. Dicha verificación de los elementos fueron realizados por el EHEO8 ${ }^{8}$, el cual maneja valores en factores de seguridad muy parecidos a los del manual de acero LRFD, por lo que no presenta ningún problema.

Ya que el Eurocódigo maneja factores de seguridad muy altos, en México podrá ser aplicado sin ningún problema puesto que estos son mucho más bajos. Por otro lado el impacto económico sería muy alto, pero para nuestra investigación emplearemos la normativa que el programa ha manejado.

8 Se refiere al método de fuerzas desequilibrantes. Se coloca una carga en un punto de un sistema estructural para desequilibrarlo, se genera un sinfín de ecuaciones de segundo orden, y en consecuencia buscan la posición equilibrada por medio de matrices que generan iteraciones hasta encontrar el modelo itinerante que equilibre dicho sistema. Fuente: http://tecno.upc.edu/ wintess/manual/
Volviendo a nuestro análisis, observamos que las barras con mayor esfuerzo de momento y tensión, no superan el Ratio de seguridad establecido a la unidad (Tabla 2). Debemos mencionar que para el tipo de estructura que analizamos, la comprobación sigue siendo a la unidad 1 pero por comprobación de seguridad en los elementos tubulares es de 1.65. Puesto que esta estructura no es del tipo transitable dicho método genera mucha controversia. Sin embargo el Dr. Ramón Sastre Sastre y Dr. José Ignacio Llorens Duran, especialistas en el tema, han comprobado que dichas estructuras pasan con un factor de seguridad 1.65 y ya están redactando dichas reglamentaciones en Europa.

Ya que los esfuerzos de tensión axiales son los que predominan sobre la estructura, observamos que los momentos sobre la cubierta se mantienen bajos (Figura 24). Esto se debe a que la configuración estructural de la cubierta actúa como vigas en celosía, teniendo como consecuencia que los elementos actúen en su máxima capacidad diagonalmente. Aun así, aquí notamos que los axiales aumentaron como consecuencia de la presión aplicada por el viento a la membrana por lo que debemos aumentar la dimensión de las barras. 
Tabla 2

Datos de Barras Rígidas. Se observa que los esfuerzos a considerar siguen siendo los axiles, ya que el sistema funciona como celosía en toda la cubierta.

\begin{tabular}{|c|c|c|c|c|c|c|c|}
\hline Barra & Axial & Torsor & M.max & C.max & Tensión & Ratio & \\
\hline & $\mathrm{T}$ & $\mathrm{Tm}$ & $\mathrm{Tm}$ & $\mathrm{T}$ & $\mathrm{kg} / \mathrm{cm}^{2}$ & & \\
\hline 1977 & -15.190 & 0.028 & 0.180 & 0.013 & -776.2 & 0.89 & Ø150.5_S235 \\
\hline 1985 & 14.149 & 0.015 & 0.224 & 0.018 & 1267.6 & 0.89 & Ø150.5_S235 \\
\hline 1993 & -20.120 & 0.026 & 0.233 & 0.014 & -1047.9 & 1.16 & Ø150.5_S235 \\
\hline 2066 & 15.497 & 0.015 & 0.214 & 0.026 & 1349.6 & 0.95 & Ø150.5_S235 \\
\hline 2067 & -9.990 & 0.017 & 0.282 & 0.017 & -332.3 & 0.71 & $\varnothing 150 \cdot 5 \_S 235$ \\
\hline 2074 & -16.809 & 0.026 & 0.211 & 0.004 & -845.1 & 0.99 & $\varnothing 150 \cdot 5$ Ø235 \\
\hline 2090 & -14.778 & 0.028 & 0.174 & 0.005 & -754.5 & 0.86 & Ø150.5_S235 \\
\hline 2175 & 6.870 & 0.003 & 0.948 & 0.082 & 1292.9 & 0.91 & $\varnothing 150 \cdot 5 \_S 235$ \\
\hline 2184 & 6.857 & 0.003 & 0.936 & 0.081 & 1281.5 & 0.90 & Ø150.5_S235 \\
\hline 2454 & -17.473 & 0.000 & 0.181 & 0.074 & -933.2 & 0.98 & Ø150·5_S235 \\
\hline 2462 & 19.036 & 0.002 & 0.252 & 0.123 & 1371.3 & 0.96 & Ø150·5_S235 \\
\hline 2467 & 16.540 & 0.003 & 0.189 & 0.077 & 1373.9 & 0.96 & Ø150.5_S235 \\
\hline 2469 & -16.634 & 0.007 & 0.253 & 0.123 & -646.4 & 0.89 & Ø150.5_S235 \\
\hline 2474 & -19.051 & 0.007 & 0.250 & 0.122 & -779.4 & 0.99 & Ø150.5_S235 \\
\hline 2475 & -18.894 & 0.024 & 0.108 & 0.009 & -945.4 & 0.86 & Ø150.5_S235 \\
\hline 2485 & -16.843 & 0.009 & 0.266 & 0.129 & -651.0 & 0.90 & Ø150.5_S235 \\
\hline 2502 & 16.507 & 0.004 & 0.194 & 0.079 & 1373.5 & 0.96 & Ø150.5_S235 \\
\hline 2505 & -17.294 & 0.000 & 0.175 & 0.072 & -925.2 & 0.97 & Ø150.5_S235 \\
\hline 2507 & 13.091 & 0.018 & 0.260 & 0.028 & 1205.2 & 0.85 & Ø150.5_S235 \\
\hline 2509 & -18.468 & 0.008 & 0.265 & 0.129 & -738.6 & 0.97 & Ø150.5_S235 \\
\hline 2512 & 18.782 & 0.005 & 0.216 & 0.105 & 1324.0 & 0.93 & Ø150.5_S235 \\
\hline 2513 & 16.938 & 0.033 & 0.177 & 0.086 & 1223.9 & 0.86 & Ø150.5_S235 \\
\hline
\end{tabular}




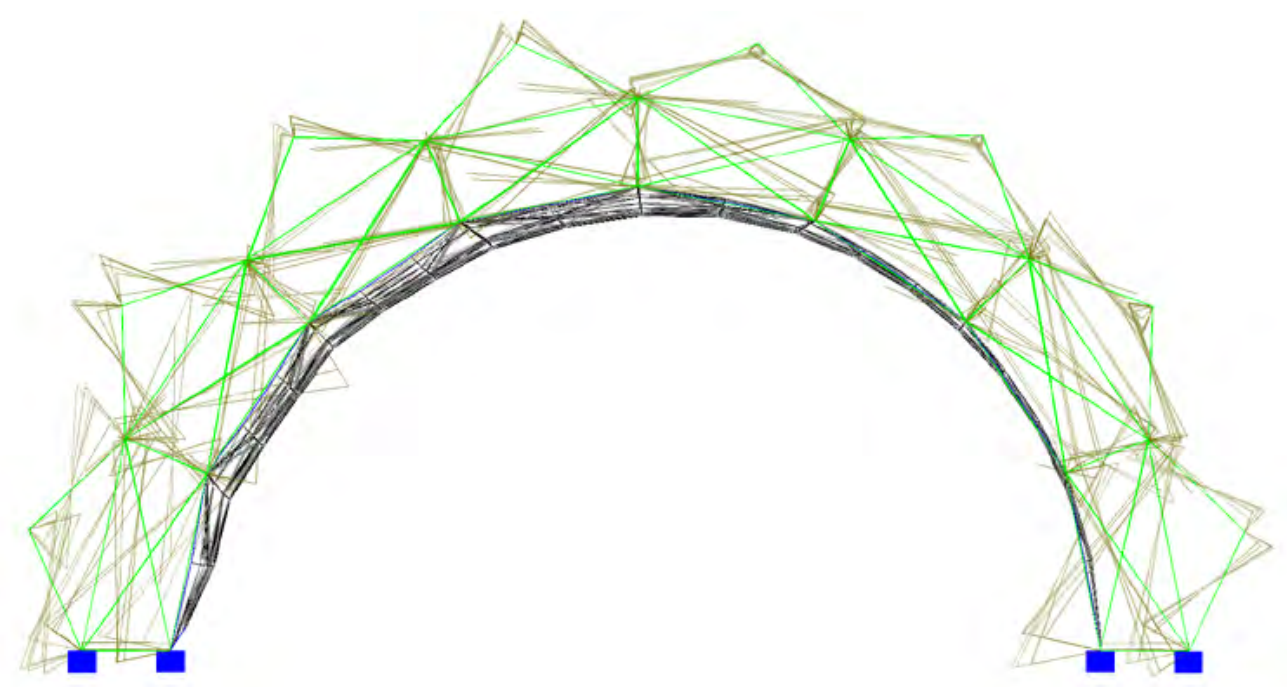

Figura 24. Ya que la figura de tijera funciona como una celosía y gracias a la disposición de los elementos de la estructura, los momentos de la cubierta son muy bajos.

Fuente: Morales, 2012

De acuerdo con la serie de análisis que ha arrojado el programa, podemos verificar la seguridad estructural del cable y la membrana textil. Estos dos elementos trabajan solo a tracción por lo que debemos tener cuidado de cuanta tensión podrían soportar de acuerdo a las propiedades del material y su módulo de elasticidad (Tabla 3).

Recordemos que se han propuesto cables con espesor de $40 \mathrm{~mm}$ y $32 \mathrm{~mm}$, y que nos dio un margen de seguridad sobrado pero que así manejaremos para efectos del presente ejercicio. las cubiertas, ya que de ellas depende la viabilidad del proyecto. La membrana Serge Ferrari-Fluitop-T2-1002, soporta perfecta las deformaciones causadas por las presiones de empuje y succión del viento. Con estos datos podemos diseñar las conexiones reales para nuestra cubierta plegable y mejorar las uniones y detalles de fijación (Figura 25).

La finalidad de realizar el análisis con ayuda de este programa, fue el de obtener las especificaciones de diseño de las conexiones de la cubierta, así como el de conocer el esfuerzo real de la estructura sobre la membrana. 
Tabla 3.

En la tabla podemos verificar las dimensiones que se deben de contemplar para el diseño constructivo de las conexiones de la membrana y la estructura.

\begin{tabular}{ccccc}
\hline \multicolumn{5}{c}{ TRACCIÓN MAXIMA EN LA MEMBRANA } \\
\hline Barra & Nudos & T/metro & $\mathrm{kg} / 5 \mathrm{~cm}$ & Ratio \\
\hline 1728 & $487-519$ & 1.470 & 73.5 & 0.90 \\
\hline \multicolumn{5}{c}{ TRACCIÓN EN LOS CABLES DE RELINGA } \\
\hline Cable & $\mathrm{T}$ & Ratio & Barra \\
\hline 1 & 2.860 & 0.06 & $1,2,3,4,5,6,7,8,9,10,11,12,13,14,1 ?$ WS-2-(40mm)Galv \\
2 & 2.857 & 0.06 & $45,46,47,48,49,50,51,52,53,54,55,5 ?$ WS-2-(40mm)Galv \\
3 & 1.593 & 0.07 & $65,66,67,68,69,70,71,72,73,74,75,7 ? 1 \times 91-(32 \mathrm{~mm})$ Inox \\
\hline
\end{tabular}

Fuente: Morales, 2012.

Ratio

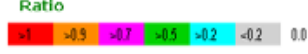

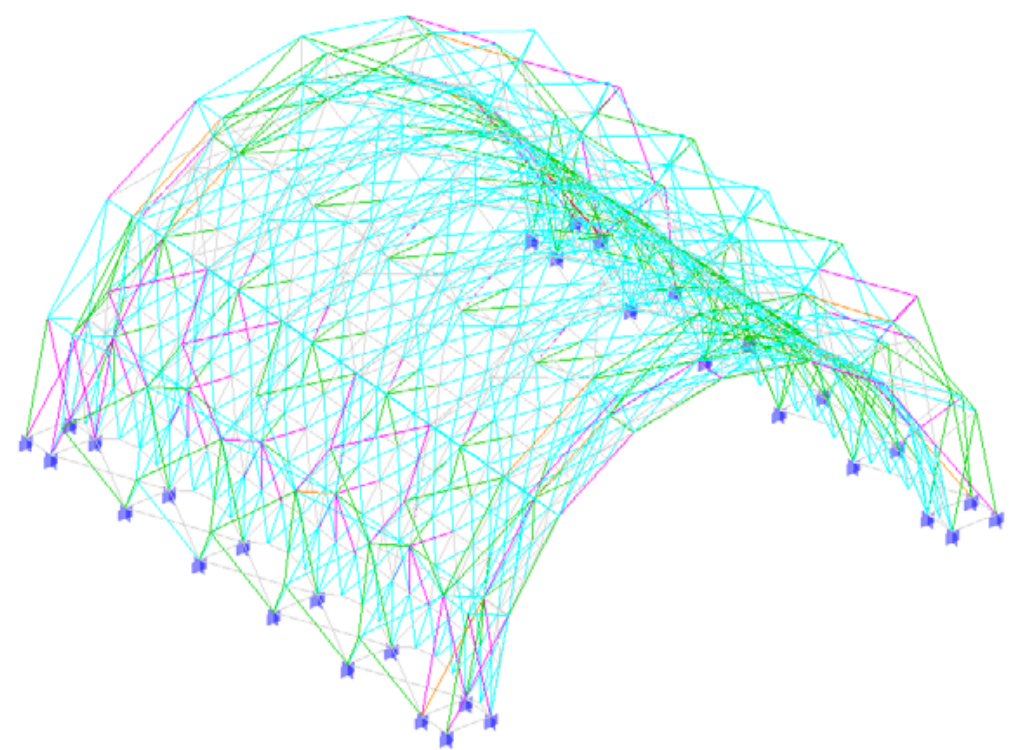

Figura 25. En la figura podemos observar que el Ratio de seguridad de los tubos, cables y la membrana no sobrepasa a los establecidos. Factor de seguridad excedente es de color rojo. 

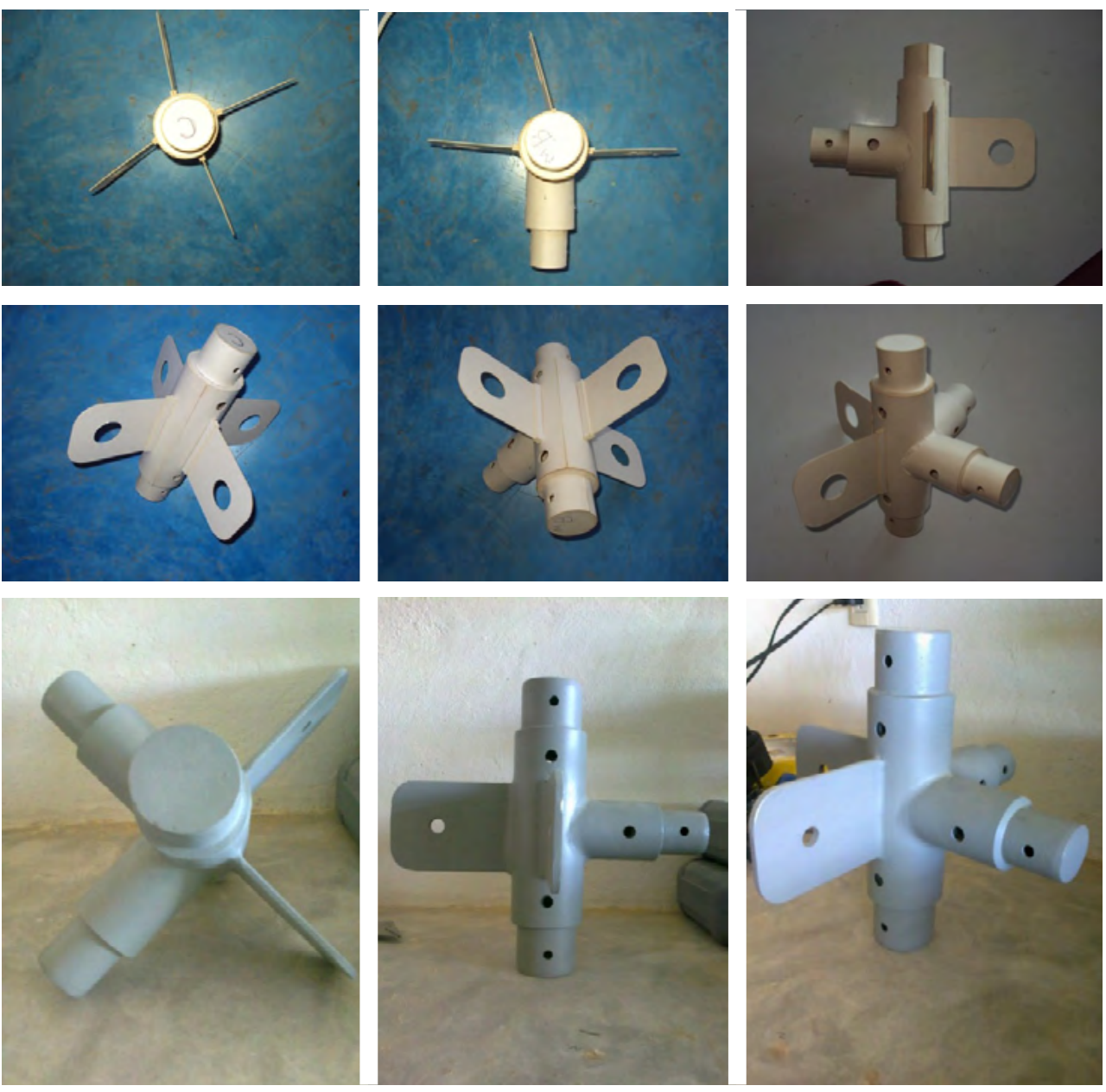

Figuras 26 - 34. Desarrollo del Modelo experimental del nodo tipo. Fuente: Morales, 2012.
Específicamente logramos definir las dimensiones reales para la estructura propuesta de nuestro proyecto final que es una cubierta plegable. Es importante mencionar que para ello no se han realizado las comprobaciones de conexión por elemento finito como en caso del prototipo. Como este sistema será patentado una vez completada la propuesta, dichas comprobaciones se harán en una segunda etapa de la investigación. Así pues se derivará un prototipo mejorado con las conexiones ya corregidas y verificadas para su funcionamiento óptimo dentro de la estructura plegable.

\section{Ejemplar del Prototipo}

Evidencia Fotográfica del Modelo y su Montaje.

- Desarrollo del Diseño del Nodo Tipo.

En esta etapa se construyó un modelo aproximado del nodo tipo, escala 1:1 de papel batería. Este prototipo del nodo se envió con un herrero experimentado en el área para que el posteriormente lo fabricara a una misma escala pero esta vez a base de tubería de acero con un espesor de $89 \mathrm{~mm}$. Este nos servirá de guía para la corrección del nodo y posteriormente se producirá en serie para los 8 módulos de la cubierta pegable (Figura 26-34). 
Se han corregido dos aspectos muy importantes:

1. De acuerdo a la experimentación con el prototipo se llegó a la conclusión de que tendrá que ser un nodo compuesto. Así pues tendrá la característica de adaptarse al sitio siendo estructuralmente más eficiente. La estructura debe entrar en la etapa de plasticidad pero no de ruptura, lo cual significa que es un sistema de segundo orden ya que es rigidizada y estabilizada por cables y una membrana mediante esfuerzos de tracción y compresión.

2. La forma más viable es que fuese construido mediante un vaciado monolítico, pero por la falta de recursos monetarios, se hará por piezas compuestas de tubos y placas.

Podemos mencionar que tras la generación del modelo en la presente etapa de la investigación, concluimos con el proceso de pre-diseño del nodo tipo. A continuación se dejan las estrategias y proceso para la construcción de la cubierta pegable con el uso del nodo estándar.

\section{Resultados de Construcción del Prototipo}

\section{Primera Prueba de Montaje}

La intención de esta primera prueba es la de comprobar que las uniones y los demás elementos empalmaran perfectamente entre sí y poder obtener la geometría de la estructura calculada. De no ser así, se podrá corregir con anticipación cualquier error que presente.

La estructura, al ser montada a la intemperie, estará sometida a grandes niveles de humedad $(65 \%$ a $85 \%$ de humedad relativa) característicos de la región, además de interactuar con otros contaminantes como el salitre y componentes químicos por ser una zona petrolera. En primera instancia, se aplicó una capa primaria de Primer a los elementos estructurales y nodos, para luego aplicar una pintura epopxica que evitará la oxidación de la estructura.

La estrategia para armar la estructura plegable es colocar cada uno de los elementos de tal manera que los nodos superior e inferior se encuentran ya sujetados por los rigidizadores. 


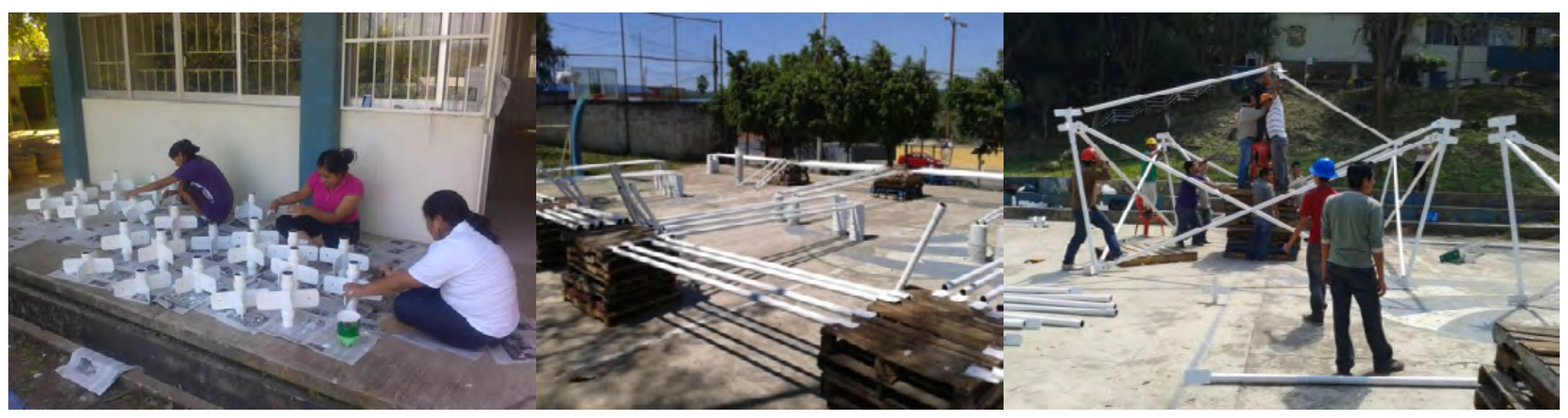

Figuras 35 - 37. Proceso de la primera prueba de montaje de la estructura plegable.

Fuente: Morales, 2012.

La unión de estos es por medio de tornillos y tuercas de $1 / 2$ " $\varnothing$ estabilizando el módulo de la cubierta parcialmente. A continuación se realiza la colocación de los elementos en tijera en una posición arqueada y se procede a atornillar los nodos junto con sus sujeciones. En seguida se une este módulo con las tijeras lineales para así formar la geometría del módulo cuadriculado.

Ya formado el modulo, se montaron algunas tarimas al centro de toda la estructura como auxiliares en la colocación de los elementos de contra venteo en los cuatro vértices. Estos van sujetos al nodo de contra venteo fijados con tornillos de la misma especificación utilizada para el modulo. La estructura se estabiliza parcialmente gracias a su mismo peso (Figura 35-37).
Los 8 módulos que conforman a la estructura plegable se arman de manera independiente y de esta manera detectar algún error de fabricación de las piezas. Posteriormente estos se ensamblan en pares y con ayuda de las tarimas, se genera una contra flecha que sostiene la estructura parcialmente. Por último se realiza la misma operación de montaje de los accesorios de conexión formando la geometría curva de la estructura. No se observó problema alguno durante esta prueba de montaje.

Realizar el montaje parcial de la estructura nos abre un panorama de los posibles errores y técnicas que deberán corregirse durante el montaje real. Además se han establecido nuevas estrategias en su construcción 


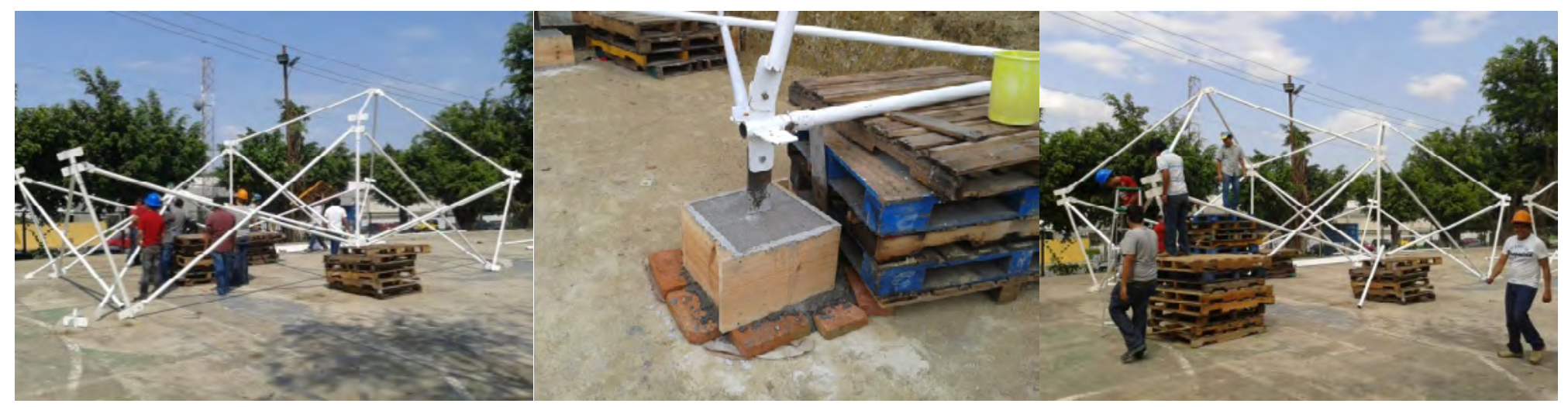

Figuras 38 - 40. Proceso de ensamblaje de los módulos y construcción de basamento de concreto simple para cada extremo de la estructura.

Fuente: Morales, 2012

que permiten que la estructura tenga mayor estabilidad. Una de ellas es que se colocarán en la parte inferior de la estructura, accesorios para la conexión con cables para arriostrar cada uno de los módulos. También se ha decidido construir basamentos de concreto para nivelar y soportar los extremos de la estructura plegable. Las especificaciones empíricas para dichos basamentos: dimensiones de $0.50 \times 0.50 \times 0.60 \mathrm{~m}$ y armados con variIlas $3 / 8 " \varnothing$.

Es vital mencionar que a pesar de que lo ideal del montaje es que sea realizado por medio de una grúa, debido a los tiempos, para efectos de la etapa de demostración se realizará por andamiaje. Aún queda por realizar algunas correcciones de los elementos y nodos, pero gracias a la geometría del sistema, la estructura plegable se encuentra totalmente estable.

\section{Segunda Prueba de Montaje.}

El objetivo fundamental es el de montar por completo el arco de la estructura plegable, ya incluidas sus conexiones fijas y atornilladas. El método utilizado es por armado de las conexiones rígidas. Por el bajo presupuesto asignado para este proyecto, no se pudieron fijar las tijeretas y nodos para que fueran desplegadas por una grúa por lo que se armaron como piezas espaciales. De esta manera, el izaje llevaría menos tiempo y por lo tanto la renta de la grúa sería más bajo. 


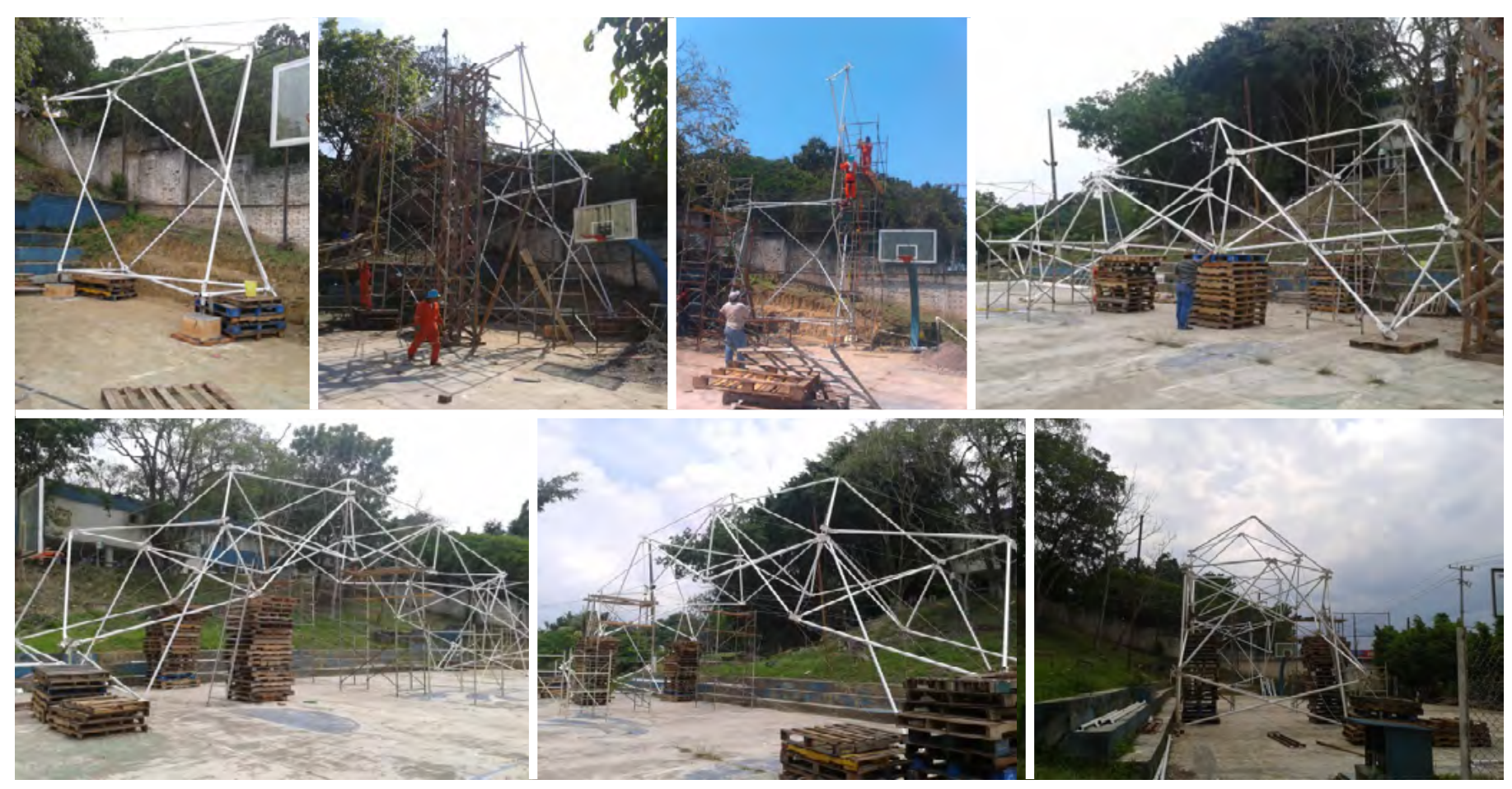

Figuras 41 - 47. Proceso de montaje para la arco de la estructura plegable conformada por 8 módulos. Fuente: Morales, 2012.

En esta ocasión se unieron 5 módulos de manera continua formando una sola pieza para que enseguida, con ayuda de la grúa, esta fuese colocada sobre otros dos módulos: uno en cada extremo del arco. Primero se conecta, en cada uno de los extremos, el módulo de tijeretas para que de esta manera los 6 módulos queden en una sola pieza. De acuerdo al proyecto, el arco debería estar conformado por 8 módulos pero hasta el momento por motivos presupuestarios, no se ha logrado completar dicha pieza. Este arco tiene una contra flecha inferior de $12.50 \mathrm{~m}$ y en la punta más alta de 14.50m (Figura 41-47). Al no encontrarse arriostrada la parte inferior de la estructura, los extremos comenzaron a pre-reflectarse. Para contrarrestar dichos esfuerzos, se colocaron cables arriostrados y de esta manera obtener una pieza perfecta para el izaje de la estructura. 
Tras esta segunda prueba de montaje podemos mencionar tres puntos importantes:

1. El peso de los elementos y nodos que forman el arco de la estructura no permite realizar maniobras constructivas en un menor tiempo, y dado que es una estructura transformable debería permitir acortar los tiempos de edificación.

2. Deberá replantearse el diseño del nodo puesto que en este ejercicio presentó problemas de excentricidad en las uniones que se conectaron, y aunque no presentó ninguna deformacion, no en todos las partes de la estructura se conectaron de manera exacta.

3. La colocación de la membrana en la parte inferior de la estructura plegable para que de esta manera se pueda arriostrar el diafragma debajo de sistema.

Todas estas recomendaciones deben tomarse en consideración para una mejor aplicación tecnológica constructiva del sistema estructural.

\section{Alcances y Mejoras}

Como resultado del anterior análisis, se han diseñado detalles estructurales en los que se ha demostrado que los nodos de la estructura deberán ser articulados en dos direcciones y de esta forma optimizar la plegabilidad del sistema.

En los nuevos modelos se observa que los nodos están compuestos por un nodo central articulado unido mediante tuercas y un bushing de espesor 1" y de esta manera facilitar el giro de la articulación. Siendo así, los elementos conectados podrán plegarse de una manera más fácil. En la parte superior se encuentra conectado con otro nodo compuesto que forma la figura de contra venteo. En este caso los nodos y contra venteos de unión conectados se componen por un nodo esférico de cuatro brazos; esto con la finalidad de que tenga un tope que sujete y también gire sobre su eje. Se sujeta un atiezador en la parte inferior de los nodos principales que es el que estabiliza y rigidiza a la estructura; por otro lado los nodos principales de la parte inferior se han colocado sujetadores de pretensado que los mantienen unidos con la membrana (Figura 48-51). 

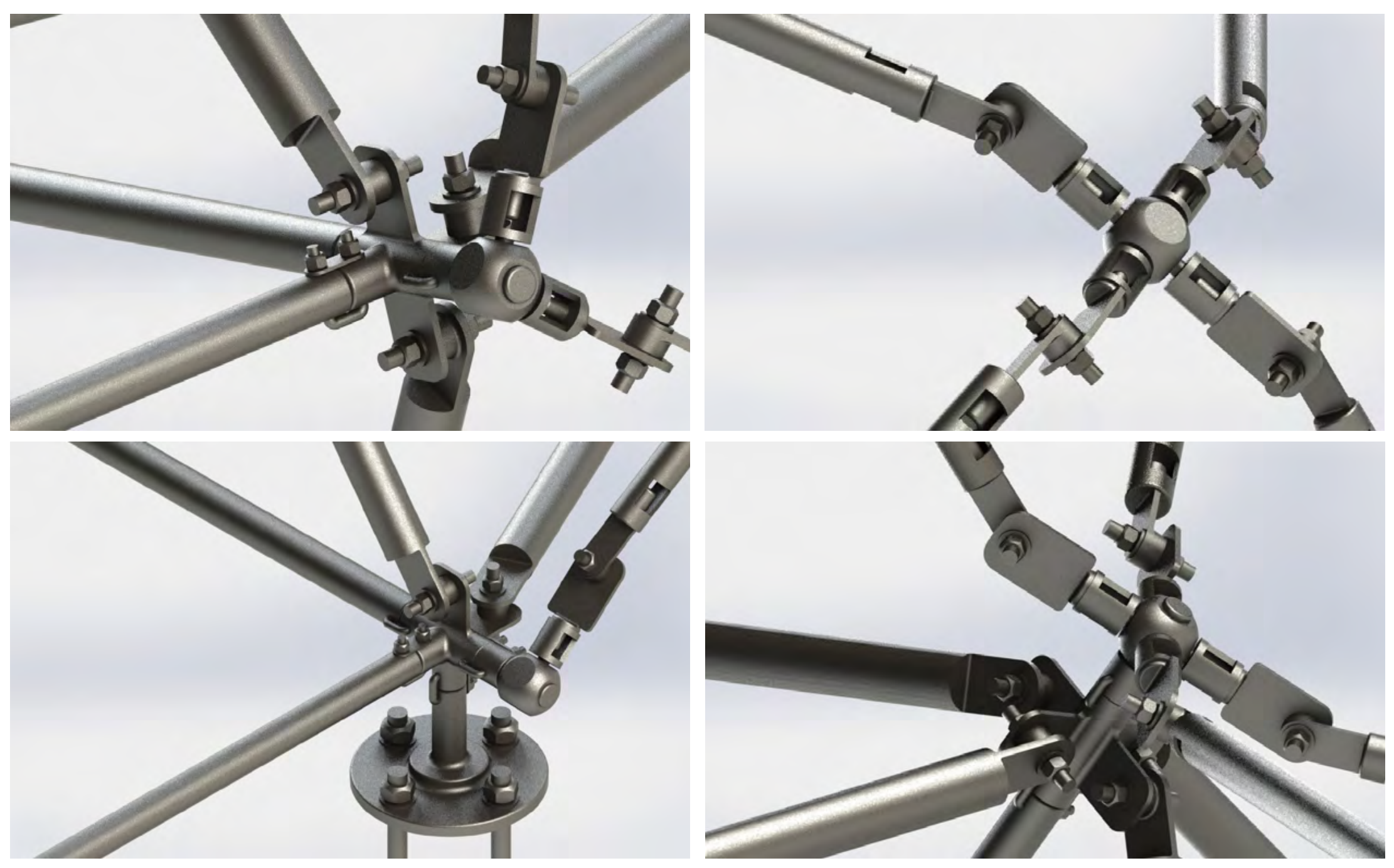

Figuras 48 - 51. Se desarrollaron los detalles de la estructura en un modelo 3d, para que en la siguiente etapa que es la construcción, fueran solucionados de esta forma. Con ello comprobamos la traslación del diseño a la ingeniería de simulación estructural. Aunque falta comprobar los detalles en el ámbito de la construcción, la relación con la realidad siempre estuvo presente en la elaboración de estos modelos.

Fuente: Morales, 2012. 

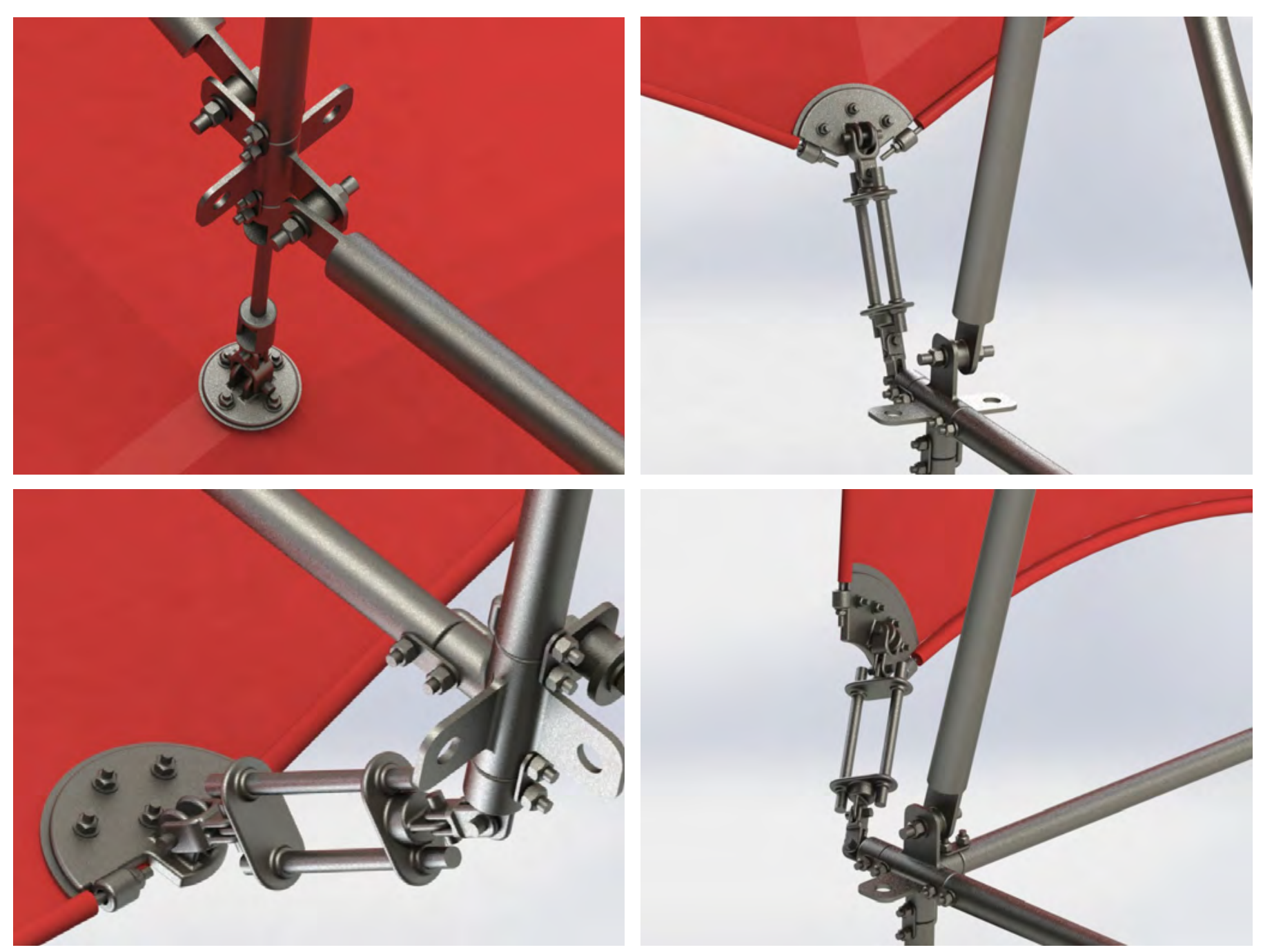

Figuras 52 - 55. Detalles de uniones de la membrana. La importancia de estos fueron la colocación del pretensado y el accesorio de bi-articulacion para sostener la flexibilidad adecuada para el equilibrio de la cubierta.

Fuente: Morales, 2012 
De igual manera se han diseñado los detalles para sujeción de la membrana de la cubierta plegable. Estas uniones se componen de un bastón pretensado que regula los esfuerzos de la cubierta. Cuenta con un accesorio bi-articulador que dota de movimiento en dos direcciones a las conexiones, estabilizando a la estructura aún si esta sufre movimientos por la fuerza del viento. Por otro lado el pretensando auxilia en la estabilización de la geometría que pudiera verse afectado por la fuerzas de tensión causados por la membrana (Figura 52-55).

\section{Conclusión}

Mediante el estudio de la tecnología estructural y con el objetivo de la industrialización del espacio, se ha desarrollado la presente investigación teniendo como principio fundamental una estructura transformable. Para lograrlo se ha implementado un sistema plegable que dota de flexibilidad a la estructura, adaptabilidad y versatilidad en cualquiera que sea el contexto. Para el diseño de dicho sistema se elaboró un nodo mediante el cual se unirían los elementos estructurales, garantizando flexibilidad y resistencia a la estructura plegable.
Esta perspectiva conceptual de diseño se encuentra justificada en las tendencias tecnológicas encontradas en la línea histórica de los sistemas transformables. Los principios básicos que han dejado estos referentes históricos pueden ayudar a reforzar la metodología de diseño realizada en la presente investigación. Podemos mencionar entonces tres principios básicos que toman como premisa el diseño de una estructura plegable y que dieron sin duda origen a esta obra: la flexibilidad de las chozas Beduinas, la prefabricación implementada por el Arq. Emilio Pérez Piñeiro y la transformabilidad como en las construcciones del Dr. Félix Escrig.

Así mismo la investigación teórica sobre la hipótesis para realizar un sistema transformable fue un factor significativo para una primera aproximación conceptual. Dichas aportaciones teóricas-prácticas se tomaron como un principio formal en la metodología para la adecuación geométrica y de esta forma construir las estructuras plegables. Se han generado otras posibles hipótesis de desarrollo geométrico comparándolas con propuestas aproximadas de conexiones y nodos, no olvidando que se requiere seguir con los estudios sobre el tema y hacer otras posibles aportaciones. 
Sin embargo, importante es el hecho de haber fabricado el módulo geométrico escala 1:1. Este nos ha permitido visualizar de una manera más real los inconvenientes de estos sistemas estructurales, y en concreto de nuestro proyecto; ahora se pueden corregir los problemas estructurales para que el prototipo final tenga una función óptima. Este además puede servir para la construcción de espacios multifuncionales y dar versatilidad a los espacios interiores.

El sistema analizado en la presente investigación, puede desarrollarse de distintas formas, pero por cuestiones prácticas solo se ha profundizado en el principio de diseño de una de las variantes y en el análisis de las propiedades físicas de sus elementos constructivos para conocer si se encuentran dentro de los rangos de seguridad establecidos. Así también, el proceso de montaje puede retomarse para generar diferentes posibilidades al realizarlo.

En la actualidad el usuario moderno enfrenta constantes cambios en la forma de habitar un espacio relacionándolo siempre con el concepto de temporalidad. Es por esto que se debe cambiar la forma de ver el espacio y entender que las construc- ciones rígidas hoy en día ya no son la respuesta para la satisfacción de las necesidades del individuo. Aunque no todo está solucionado en esta investigación, pues existen puntos que tendrán que estudiarse con profundidad, este puede dar la pauta para la generación de nuevas líneas de investigación. Tal es el caso de la adaptación de los sistemas bidireccionales o el desarrollo de la geodésica rebajada plegable.

\section{Referencias}

Arnal, L. y Betancourt, M. (2008). Reglamento de Construcción del Distrito Federal. México D.F. : Editorial Trillas.

IMCA - Instituto Mexicano de la Construcción en Acero. (2005). Manual de construcción en acero. Diseño por esfuerzos permisibles. México D.F.: Limusa.

McCormac, J. (2000). Diseño de Estructuras de Acero, Método LRFD. México, D.F.: Editorial Alfa omega.

Moore, F. (2000). Compresión de las Estructuras en la arquitectura, Editorial Interamericana McGrawHill, Traducción en México. 
Morales, C. (2010). Sistemas de diseño para la vivienda. Revista de Arquitectura, 13(1), 118-127.

Morales, C. (2009). Diseño de Sistemas Estructurales Flexibles en el Espacio Arquitectónico. México: Universidad Nacional Autónoma de México.

Morales, C. (2012). Diseño de Sistemas Flexibles en el Espacio Arquitectónico. España: Editorial Academia Española.

Morales, C. (2012). Diseño de una Cubierta Retráctil Tensada, Actividad Post Doctoral. España: Universidad Politécnica de Catalunya.
Morales, C. (2013). Diseño y Análisis de Sistemas Transformables en las Cubiertas Tensadas, Actividad Post Doctoral. España: Universidad Politécnica de Catalunya.

Morales, C. (2013). Diseño y Sistemas de Cubiertas Plegables Tensadas Transformables. España: Universidad Camilo José Cela.

Rodríguez, N. (2005). Diseño de una estructura transformable por deformación de una malla plana en su aplicación a un refugio de rápido montaje. Barcelona: Universidad Politécnica de Catalunya.

Segui, W. (2000). Diseño de Estructuras de Acero con LRFD. México: Editorial Thomson. 